\title{
The IST spectral portraits of the first order doubly periodic solutions of the nonlinear Schrödinger equation
}

\author{
N. Akhmediev \\ Department of Theoretical Physics, Research School of Physics, \\ The Australian National University, Canberra ACT 2600, Australia \\ J. M. Soto-Crespo \\ Instituto de Óptica CSIC, Serrano 121, 28006 Madrid, Spain
}

\begin{abstract}
The spectra of the inverse scattering technique (IST) play a crucial role in the physics of nonlinear phenomena. They define the long term evolution of dynamical systems. We present the IST spectral portraits for the extensive three-parameter families of the first order doubly periodic solutions of the nonlinear Schrödinger equation that cover a wide range of physical phenomena such as modulation instability, rogue waves and many other problems with periodic boundary conditions. We relate these spectral portraits with the parameters of the family. We show that there are two qualitatively different types of spectral portraits. A-type spectra consist of two continuous bands: a band of purely imaginary eigenvalues within the interval $[-i, i]$ and a finite band of complex eigenvalues. On the contrary, B-type spectra possess only continuous bands of imaginary eigenvalues all located within the interval $[-i, i]$ and separated by a finite band gap. A physical interpretation of these results is given.
\end{abstract}

PACS numbers: 82.40.Bj,42.65.Sf,05.70.Ln,87.19.L-,47.20.Ky

\section{INTRODUCTION}

The inverse scattering technique is an essential tool in mathematical physics that allows us to solve initial value problems for a number of partial differential equations modeling nonlinear wave propagation in physics [1]. These include the $\mathrm{KdV}$ equation, the nonlinear Schrödinger equation [2], the Sine-Gordon equation and a multiplicity of other evolution equations [3]. An essential part of this technique is the spectral analysis that provides information about the nonlinear modes participating in the evolution. In particular, this spectrum provides information about the soliton content in a wave field. Constructing the IST spectrum for the solutions of a nonlinear equation is equivalent to the Fourier analysis in linear problems [4]. As such, the IST spectral decomposition can be used in optical engineering: telecommunications [5], signal transmission [7], optical data processing [6], rogue wave analysis [8] and in other problems of nonlinear optics [9]. The IST spectrum is directly related to the spectrum of modes in linear stability analysis of stationary periodic solutions [10]. Thus, the knowledge of the IST spectrum is important both in theory and applications.

A complete IST spectral analysis requires highly involved mathematical constructions such as algebrogeometric approaches [11], finite gap methods [12, 13], Riemann surfaces [14] and theta functions [15]. However, for practical purposes, simple solutions and numerical analysis may be sufficient in order to visualize the so called "spectral portraits" [8] of the NLSE solutions of practical interest. Such spectral portraits can be constructed for modulation instability [16], rogue waves [8], integrable turbulence [17], super-regular breathers
[18] and other nonlinear phenomena common to various branches of physics.

Periodic solutions of the NLSE are an important class of solutions in physics. In principle, they can be written explicitly in the form of a quotient of theta functions [15]. However, this representation did not find many applications since such form has an infinite number of free parameters. This general form can be compared to multisoliton solutions with an infinite number of solitons in the solution. When dealing with such solutions, we have to investigate first their fundamental components. One soliton solution is presently well known. However, the fundamental components of periodic solutions are still not completely investigated.

These fundamental components are first order doubly periodic solutions, firstly presented in [19] and more recently elaborated in [20]. They are three-parameter families in the form of nontrivial combinations of Jacobi elliptic functions. Free real parameters provide the possibility of adapting the periods and the amplitude of the solution to particular physical conditions. Moreover, there are two types of these families, which provide even more flexibility.

The physical spectra of the doubly periodic solutions have been calculated in [20]. They are important to compare with the spectral measurements observed in the experiments. However, the IST spectra are fundamentally different. These are crucial components of the mathematical theory and the physical interpretation. The IST spectra are important for understanding the long-term field evolution and for building up more complex multiperiodic solutions. Such spectra have never been produced in previous works. We fill this gap in the present manuscript, thus opening ways for further progress in this area. 
In adimensional units, the nonlinear Schrödinger equation reads,

$$
i \psi_{z}+\frac{1}{2} \psi_{t t}+|\psi|^{2} \psi=0
$$

The first-order doubly periodic solutions can be written in complex form:

$$
\psi(t, z)=[Q(t, z)+i \delta(z)] e^{i \phi(z)},
$$

where $Q, \delta$ and $\phi$ are real functions, $\delta$ and $\phi$ depending only on the $z$-variable. Eq.(2) represents the lowest (first) order doubly periodic solutions of the NLSE [19]. This family of solutions can be subdivided into two groups: A- and B-type solutions [20].

Let us start with A-type solutions. This family depends on three real parameters $\alpha_{3}>0, \rho, \eta$. The function $\delta(z)$, is given by:

$$
\delta(z)=\sqrt{\frac{\alpha_{3}}{2}(1-\nu)} \sqrt{\frac{1+\operatorname{dn}(\mu z, k)}{1+\nu \operatorname{cn}(\mu z, k)}} \operatorname{sn}(\mu z / 2, k),
$$

where sn, dn and cn are elliptic Jacobi functions with modulus $m=k^{2}=\frac{1}{2}\left(1-\frac{\eta^{2}+\rho\left(\rho-\alpha_{3}\right)}{A B}\right)$. Here,

$$
A^{2}=\left(\alpha_{3}-\rho\right)^{2}+\eta^{2}, \quad B^{2}=\rho^{2}+\eta^{2}, \quad \nu=\frac{A-B}{A+B},
$$

and $\mu=4 \sqrt{A B}$. Clearly, $0<\delta^{2}<\alpha_{3}$.

The phase $\phi(z)$ is given by:

$$
\begin{gathered}
\phi(z)=\left(2 \rho+\frac{\alpha_{3}}{\nu}\right) z-\frac{\alpha_{3}}{\nu \mu}[\Pi(\operatorname{am}(\mu z, k), n, k)- \\
\left.-\nu \sigma \tan ^{-1}\left(\frac{\operatorname{sd}(\mu z, k)}{\sigma}\right)\right]
\end{gathered}
$$

where $n=\frac{\nu^{2}}{\nu^{2}-1}, \sigma=\sqrt{\frac{1-\nu^{2}}{k^{2}+\left(1-k^{2}\right) \nu^{2}}}, \operatorname{sd}(\mu z, k)=$ $\frac{\operatorname{sn}(\mu z, k)}{\operatorname{dn}(\mu z, k)}$ and $\Pi(\operatorname{am}(\mu z, k), n, k)$ is the incomplete elliptic integral of the third kind, with the argument $\operatorname{am}(u, k)$ being the amplitude function [28, 29]. The function $Q(t, z)$ is given by:

$$
Q(t, z)=s b-c_{+} \frac{r+\operatorname{cn}\left(p t, k_{q}\right)}{1+r \operatorname{cn}\left(p t, k_{q}\right)}
$$

where $s=s(z)=\operatorname{sign}[\operatorname{cn}(\mu z / 2, k)], r=\frac{M-N}{M+N}$,

$$
\begin{aligned}
& p=\sqrt{M N}=2 \sqrt[4]{\left(\alpha_{3}-\rho\right)^{2}+\eta^{2}}, \\
& k_{q}^{2}=\frac{1}{2}+2 \frac{\rho-\alpha_{3}}{p^{2}}, \quad b=\sqrt{\alpha_{3}-y}, \quad y(z)=\delta^{2}(z), \\
& c_{ \pm}=\sqrt{2\left[\sqrt{(y-\rho)^{2}+\eta^{2}} \pm(\rho-y)\right]}, \\
& M^{2}=\left(2 s b+c_{+}\right)^{2}+c_{-}^{2}, \quad N^{2}=\left(2 s b-c_{+}\right)^{2}+c_{-}^{2} .
\end{aligned}
$$
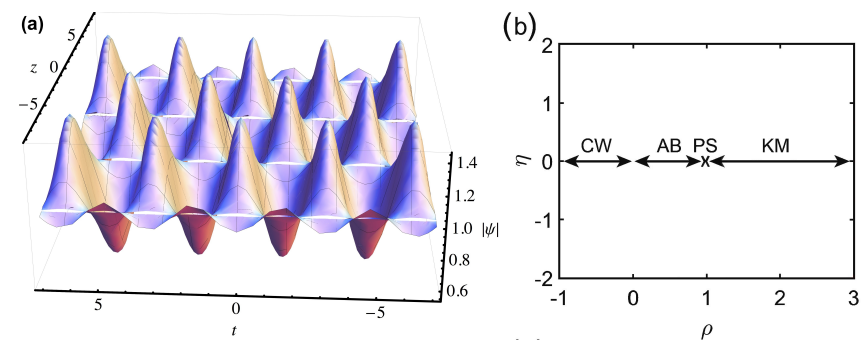

FIG. 1. (a) Evolution of a typical A-type doubly periodic solution. The corresponding parameters are $\alpha_{3}=1, \rho=0.355$ and $\eta=0.073$. The distinctive feature of these solutions is the transverse displacement of the maxima at every half of the longitudinal period. (b) Visualization of the A-type solutions in the parameter space $(\rho-\eta)$. The special solutions, located on the horizontal axis are labeled as follows: PS - Peregrine solution, CW - continuous wave, AB - Akhmediev breathers, KM - Kuznetsov-Ma solitons. Here, the parameter $\alpha_{3}=1$. Solutions are symmetric relative to the transformation $\eta \longleftrightarrow$ $-\eta$.

The periods along $z(=L)$ and $t(=T)$ are $L=$ $\frac{8 K(k)}{\mu}, T=\frac{4 K\left(k_{q}\right)}{p}$, respectively. Here $K(k)$ is the complete elliptic integral of the first kind.

The intensity profile evolution of a typical A-type solution is shown in Fig.1(a). The solution is periodic both in $z$ and $t$ with growth-decay cycles relative to a nearly $\mathrm{CW}$ state at each half-period along the $z$-axis. The maxima in the next half-period are shifted by half of a period along the $t$-axis. This shift is clearly seen in Fig.1(a). The A-type family comprises a rich variety of solutions that includes as particular cases the $\mathrm{AB}$ solutions, the KM solitons, the Peregrine solution, the bright soliton and CW solutions. The location of these sub-families on the plane of parameters $\rho$ and $\eta$ for fixed $\alpha_{3}=1$ is shown in Fig.1(b).

The family of B-type solutions has a different parameterization. Its solutions depend also on three real parameters, but now they must comply with the following condition $\alpha_{3}>\alpha_{2}>\alpha_{1}>0$. The function $\delta(z)$ is now given by:

$$
\delta(z)=\frac{\sqrt{\alpha_{1} \alpha_{3}} \operatorname{sn}(\mu z, k)}{\sqrt{\alpha_{3}-\alpha_{1} \operatorname{cn}^{2}(\mu z, k)}}
$$

where the modulus $m$ of the Jacobi elliptic functions [28, $29]$ is $m=k^{2}=\frac{\alpha_{1}\left(\alpha_{3}-\alpha_{2}\right)}{\alpha_{2}\left(\alpha_{3}-\alpha_{1}\right)}$ and $\mu=2 \sqrt{\alpha_{2}\left(\alpha_{3}-\alpha_{1}\right)}$. The function $\delta^{2}$ must be within the interval $0<\delta^{2}<\alpha_{1}$. For the function $\phi(z)$, we have:

$$
\phi(z)=\left(\alpha_{1}+\alpha_{2}-\alpha_{3}\right) z+\frac{2 \alpha_{3}}{\mu} \Pi(\operatorname{am}(\mu z, k), n, k),
$$

where $n=\frac{\alpha_{1}}{\alpha_{1}-\alpha_{3}}$ and $\Pi(\operatorname{am}(\mu z, k), n, k)$ is defined after Eq.(4). 
For the function $Q(t, z)$, we have:

$$
Q(t, z)=\frac{Q_{D}\left(Q_{A}-Q_{C}\right)+Q_{A}\left(Q_{C}-Q_{D}\right) \operatorname{sn}^{2}\left(p t, k_{q}\right)}{\left(Q_{A}-Q_{C}\right)+\left(Q_{C}-Q_{D}\right) \operatorname{sn}^{2}\left(p t, k_{q}\right)},
$$

where the modulus $m_{q}=k_{q}^{2}=\frac{\alpha_{2}-\alpha_{1}}{\alpha_{3}-\alpha_{1}}$ and $p=$ $\sqrt{\alpha_{3}-\alpha_{1}}$. Here, $Q_{C}<Q<Q_{D}$. The $z$-dependent functions $Q_{A}(z)>Q_{B}(z)>Q_{C}(z)>Q_{D}(z)$ are defined by:

$$
\begin{aligned}
& Q_{A}=s \sqrt{\alpha_{1}-y}+\sqrt{\alpha_{2}-y}+\sqrt{\alpha_{3}-y} \\
& Q_{B}=-s \sqrt{\alpha_{1}-y}-\sqrt{\alpha_{2}-y}+\sqrt{\alpha_{3}-y} \\
& Q_{C}=-s \sqrt{\alpha_{1}-y}+\sqrt{\alpha_{2}-y}-\sqrt{\alpha_{3}-y} \\
& Q_{D}=s \sqrt{\alpha_{1}-y}-\sqrt{\alpha_{2}-y}-\sqrt{\alpha_{3}-y}
\end{aligned}
$$

where $y(z)=\delta^{2}(z)$, and $s=s(z)=\operatorname{sign}\left(\delta_{z}\right)=$ $\operatorname{sign}(\operatorname{cn}(\mu z, k))$. The periods along $z(=L)$ and along $t(=T)$ axes are: $L=\frac{4 K(k)}{\mu}, T=\frac{2 K\left(k_{q}\right)}{p}$.

An example of a typical B-type solution profile is shown in Fig.2(a). All maxima of this type of solutions are located at the same values of $t$ in contrast to the Atype solutions. This is also a rich family of solutions that include, as particular cases several other sub-families, as shown in Fig.2(b).
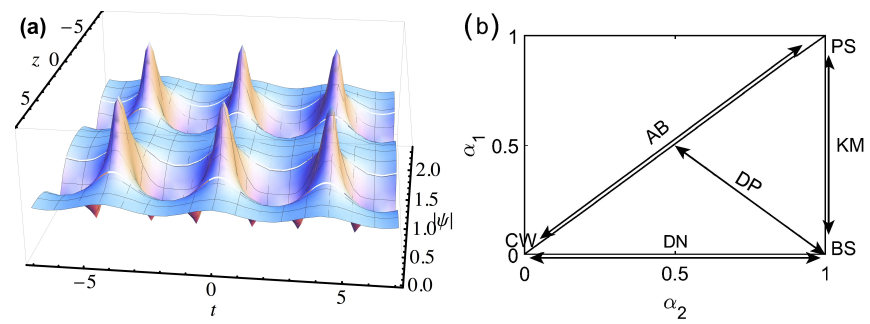

FIG. 2. (a) Typical example of a B-type solution. In contrast to the A-type solutions, there are no transverse displacements. All successive maxima remain at the same values of $t$. (b) Visualization of the B-type solutions in the parameter space $\left(\alpha_{2}-\alpha_{1}\right)$ with special solutions on the boundaries labeled as follows: PS - Peregrine solution, CW - continuous wave, AB - Akhmediev breathers, KM - Kuznetsov-Ma solitons, BS - bright soliton, DP - subclass of doubly periodic solutions given in [26], DN - stationary periodic solution given by the Jacobi elliptic function $d n$. The solutions can be symmetrically continued to the upper triangle according to the symmetry $\alpha_{1} \longleftrightarrow \alpha_{2}$. Here, the parameter $\alpha_{3}=1$.

The NLSE originates from the compatibility condition of the following set of linear matrix equations [27]:

$$
\begin{aligned}
& R_{t}=U R+\lambda J R, \\
& R_{z}=\left(\lambda^{2}+\lambda U+V / 2\right) R,
\end{aligned}
$$

where the $2 \times 2$ matrices $U, V$ and $J$ are given by

$$
U=\left(\begin{array}{cc}
0 & i \psi^{*} \\
i \psi & 0
\end{array}\right), V=\left(\begin{array}{cc}
-i|\psi|^{2} & \psi_{t}^{*} \\
-\psi_{t} & i|\psi|^{2}
\end{array}\right), J=\left(\begin{array}{cc}
i & 0 \\
0 & -i
\end{array}\right)
$$

while $R=\left(\begin{array}{c}r \\ s\end{array}\right)$ is a column matrix composed of the two complex functions $r(z, t)$ and $s(z, t)$.

It is easy to solve analytically the eigenvalue problem when the solution of the NLSE is a plane wave $\psi=A \exp \left(i A^{2} z\right)$. As we are dealing with waves of amplitude $A=1$, this solution is $\psi=\exp (i z)$. The eigenvalue problem is then:

$$
\begin{array}{r}
r_{t}=i e^{-i z} s+i \lambda r \\
s_{t}=i e^{i z} r-i \lambda s .
\end{array}
$$

Eliminating $s$, we arrive to the following linear equation:

$$
r_{t t}=-\left(1+\lambda^{2}\right) r .
$$

The solution to this equation is

$$
r=\mathcal{A} \exp \left[i\left(\sqrt{\left(1+\lambda^{2}\right)} t+\varphi\right)\right]
$$

where $\mathcal{A}$ is an arbitrary amplitude and $\varphi$ is an arbitrary phase. In order for $r$ to be a bounded function, $(1+$ $\left.\lambda^{2}\right)$ must be a real number. Therefore the eigenvalue $\lambda$ must be either a purely real arbitrary number or a purely imaginary number within the interval $[-i, i]$. This is the spectrum of eigenvalues for a plane wave solution of the NLSE. It comprises all points on the real axis $[-\infty, \infty]$ and the points on the imaginary axis within the interval $[-i, i]$. The $\mathrm{AB}$ solutions are transformed into a plane wave at $\pm \infty$. As the IST spectrum does not depend on $z$, the $\mathrm{AB}$ solutions have the same spectral portrait as the plane wave.

Strictly speaking, each solution of the $\mathrm{AB}$ family has a fixed frequency, $\omega$, that corresponds to a single imaginary eigenvalue $\lambda=i \sqrt{1-\omega^{2} / 4}$ [23]. However, the background, and consequently any $\mathrm{AB}$ solution, is unstable relative to the excitation of any of these frequencies. Thus, each AB solution has an IST spectral portrait that includes all eigenvalues within the interval $[-i, i]$. Moreover, multi-AB solutions [21] that consist of several ABs have the same continuous IST spectrum.

Each eigenvalue in the IST spectrum corresponds to the excitation of the nonlinear mode associated to this eigenvalue. The excitation of the $\mathrm{AB}$ solution at the growing stage corresponds to modulation instability. Thus, the existence of any eigenvalue within the interval $[-i, i]$ indicates the instability of the wave field that has that eigenvalue.

The doubly periodic solutions considered above are structures much more complicated than plane waves and finding their IST spectra requires the numerical solution of the eigenvalue problem given by Eq.(13). The discretisation needed for the numerical calculations transforms the continuous spectra into discrete ones. The numerical grid should cover as many periods of the solution as possible in order to approach closely a continuous spectrum of eigenvalues. For practical purposes, $100 t$-periods of the doubly periodic solutions are sufficient for finding the 
spectral curves. Nevertheless, when the period becomes too large, we have to reduce the number of periods in order to sample the wave profile with sufficient accuracy. All the results presented here were obtained using complex matrices $20000 \times 20000$. This number allows us a good compromise between the accuracy of the calculations, and the use of CPU time and memory requirements.

\section{IST SPECTRA FOR B-TYPE SOLUTIONS}

For the calculation of the IST spectra, we can use the function $\psi(z, t)$ at any arbitrary point $z$. The most convenient one is $z=0$. At this point, the functions $\delta=0$, $y=0$, and $\phi=0$, while $s=+1, Q_{A}=\sqrt{\alpha_{1}}+\sqrt{\alpha_{2}}+\sqrt{\alpha_{3}}$, $Q_{B}=-\sqrt{\alpha_{1}}-\sqrt{\alpha_{2}}+\sqrt{\alpha_{3}}, Q_{C}=-\sqrt{\alpha_{1}}+\sqrt{\alpha_{2}}-\sqrt{\alpha_{3}}$, and $Q_{D}=\sqrt{\alpha_{1}}-\sqrt{\alpha_{2}}-\sqrt{\alpha_{3}}$ are real constants. Then, $\psi(t)=Q(t)$ is a periodic function of $t$ only. Moreover, it is purely a real function. Namely, $\psi_{B}(t)=\psi(t, 0)=$

$$
=\frac{Q_{D}\left(\sqrt{\alpha_{1}}+\sqrt{\alpha_{3}}\right)-Q_{A}\left(\sqrt{\alpha_{1}}-\sqrt{\alpha_{2}}\right) \operatorname{sn}^{2}\left(p t, k_{q}\right)}{\left(\sqrt{\alpha_{1}}+\sqrt{\alpha_{3}}\right)-\left(\sqrt{\alpha_{1}}-\sqrt{\alpha_{2}}\right) \operatorname{sn}^{2}\left(p t, k_{q}\right)} .
$$

We used this function in Eq.(13) for the numerical calculation of the IST spectra. Of course, the spectra were the same for any other value of $z$.

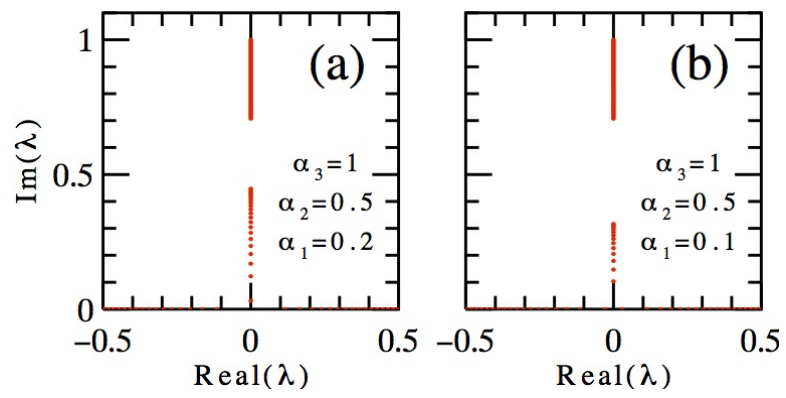

FIG. 3. Typical IST spectra of the B-type doubly periodic solutions of the NLSE for (a) $\alpha_{1}=0.2$, and (b) $\alpha_{1}=0.1$. The two other parameters are $\alpha_{2}=0.5$ and $\alpha_{3}=1$. The finite band gap on the imaginary axis appears when $\alpha_{1} \neq \alpha_{2}$. It grows as the difference between $\alpha_{1}$ and $\alpha_{2}$ increases. The discreteness in the spectrum is due to the sampling limitations of the numerical scheme.

Two examples of the IST spectra for B-type solutions found numerically are shown in Fig.3. These are calculated for the same values of $\alpha_{2}=0.5$ and $\alpha_{3}=1$, and two values of $\alpha_{1}:=0.2$ and 0.1 respectively. As the spectra are symmetric relative to the real axis, only the upper half of the complex plane is shown. All IST spectra of the B-type solutions contain only purely real and purely imaginary eigenvalues. The real eigenvalues are spread across the whole axis $[-\infty, \infty]$. They correspond to small amplitude radiative waves. All imaginary eigenvalues are located within the interval $\left[-i \sqrt{\alpha_{3}}, i \sqrt{\alpha_{3}}\right]$. The distinctive feature of these spectra is the presence of a finite band gap on the imaginary axis as can be seen in Fig.3. The size and the position of the band gap depend on the parameters $\alpha_{1}$ and $\alpha_{2}$.

We have made a systematic study of these band gaps within the IST spectrum. These results are shown in Fig.4. The red dots represent the numerically found imaginary eigenvalues while the blue dashed lines represent the curves $\sqrt{\alpha_{i}}, i=1,2$ and 3 . As it can be seen from the two plots, the boundaries of the spectral bands are given exactly by the square roots of $\alpha_{i}$. Namely, the upper limit of the spectra is given by $\sqrt{\alpha_{3}}$. The upper limit of the band gap is given by $(\operatorname{Im} \lambda)_{\max }=\sqrt{\alpha_{2}}$. The lower limit of the band gap is given by $(\operatorname{Im} \lambda)_{\min }=\sqrt{\alpha_{1}}$. Consequently, the width of the band gap $\Delta$ is $\sqrt{\alpha_{2}}-\sqrt{\alpha_{1}}$. As expected, the band gap tends to zero when $\alpha_{1} \rightarrow \alpha_{2}$ and completely disappears in the $\mathrm{AB}$ limit when $\alpha_{1}=\alpha_{2}$.
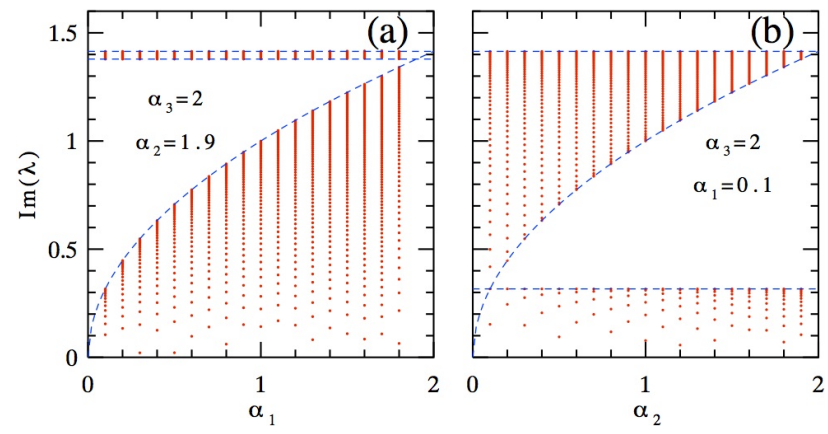

FIG. 4. Changes in the imaginary part of the IST spectra for B-type solutions as (a) $\alpha_{1}$ varies while $\alpha_{2}=1.9$ remains constant and (b) $\alpha_{2}$ varies while $\alpha_{1}=0.1$ remains constant. The three blue dashed lines inside of each panel correspond to $\sqrt{\alpha_{i}}(i=1,2,3)$. They delineate, exactly, the boundaries of the eigenvalue bands. In both cases $\alpha_{3}=2$.

The spectral components within the band gap $\left[i \sqrt{\alpha_{1}}, i \sqrt{\alpha_{2}}\right]$ on the imaginary axis correspond to a selected range of $\mathrm{AB}$ modes in the IST spectrum. The absence of these components means that the corresponding $\mathrm{AB}$ modes are incompatible with B-type solutions. In other words, these modes cannot be generated simultaneously with B-type solutions starting from any initial conditions. Thus, the B-type solution suppresses these modes despite the presence of a CW background with average amplitude $\sqrt{\alpha_{3}}$. This happens due to the parametric interaction of nonlinear modes which can be considered as a generalization of the ideas developed in the case of finite dimensional systems [22]. The NLSE describes systems with an infinite number of degrees of freedom and the set of modes is more complicated. Nevertheless, the band gaps have a similar structure and that justifies the analogy. 


\section{IST SPECTRA FOR A-TYPE SOLUTIONS}

For the A-type solution, at $z=0 \delta=0$ and $\phi=0$, while $s=+1, y=0, b=\sqrt{\alpha_{3}}$, and $c_{ \pm}=$ $\sqrt{2\left[\sqrt{\rho^{2}+\eta^{2}} \pm \rho\right]}$ are constants. Then, $\psi(t)=Q(t)$ is a real function:

$$
\psi(t, 0)=b-c_{+} \frac{r+\operatorname{cn}\left(p t, k_{q}\right)}{1+r \operatorname{cn}\left(p t, k_{q}\right)}
$$

Using it in the eigenvalue problem (Eq.13), we obtain the IST spectrum. Figure 5 shows a typical IST spectral portrait calculated for an A-type doubly periodic solution. The spectrum consists of 3 branches: a branch of complex eigenvalues with $\operatorname{Im} \lambda>1.1$, a branch of purely imaginary eigenvalues filling the interval $[-i, i]$, and the radiation band with purely real eigenvalues. The latter is always present in the IST spectra and it is not shown in Fig.5. All three bands are continuous. Again, the discreteness in Fig.5 is related to the finite number of mesh points in the numerical scheme. Here, the spectral bands do not intersect. However, this is not always the case.

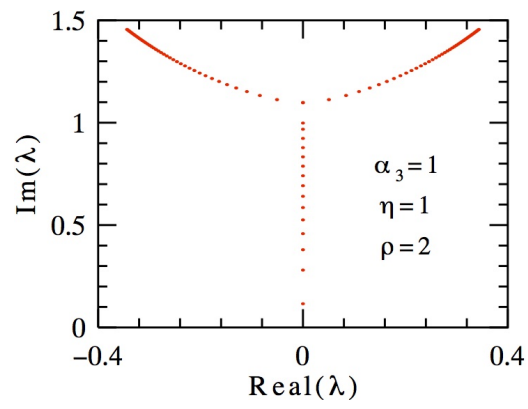

FIG. 5. IST spectrum for the A-type doubly periodic solution of the NLSE corresponding to $\rho=2$ and $\eta=1$ and $\alpha_{3}=1$.

The distinctive feature of the IST spectra of the A-type solutions with respect to the spectra of the B-type ones is the band of complex eigenvalues with both real and imaginary parts different from zero (except in its minimum). It can be approximated by a parabola with its vertex located on the imaginary axis. In the particular case, shown in Fig.5, the vertex of the parabola is located above the point $i$ that limits the branch of imaginary eigenvalues. It stays on the imaginary axis but moves up or down when parameters $\rho$ and $\eta$ change. When moving down, it can intersect the branch of purely imaginary eigenvalues. The branches of purely real and purely imaginary eigenvalues remain fixed for any values of the parameters $\rho$ and $\eta$. Only the branch of complex eigenvalues moves up and down when these parameters are changed. This branch has not only a lower limit but also an upper limit, that depends on the solution parameters. In Fig.5, this limit is around $i 1.46$.

The pattern of changes of the parabolic branch with the change of parameters can be seen from Fig.6. Here,
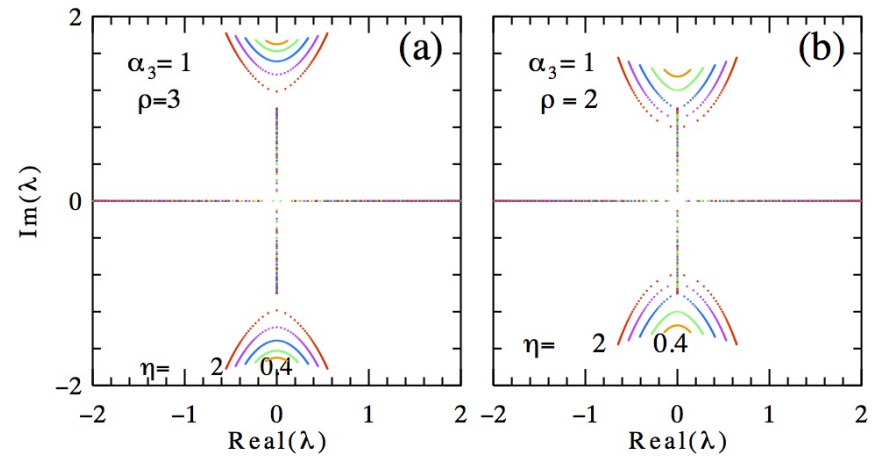

FIG. 6. IST spectra of five A-type doubly periodic solutions when $\alpha_{3}=1$, (a) $\rho=3$ and (b) $\rho=2$. In both panels, the parameter $\eta=2$ (red dots), $\eta=1.6$ (violet dots), $\eta=1.2$ (blue dots), $\eta=0.8$ (green dots) and $\eta=0.4$ (orange dots). Only the branch of complex eigenvalues varies with $\eta$. Its size reduces when $\eta$ approaches zero. It can intersect the branch of purely imaginary eigenvalues $[-i, i]$ as it can be seen in (b).
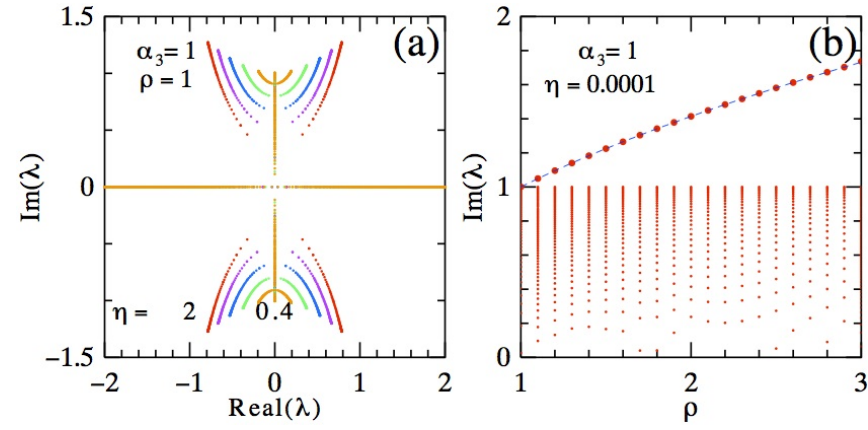

FIG. 7. (a) IST spectra of five A-type doubly periodic solution corresponding to $\alpha_{3}=1, \rho=1$, and $\eta=2($ red dots $), \eta=$ 1.6 (violet dots), $\eta=1.2$ (blue dots), $\eta=0.8$ (green dots) and $\eta=0.4$ (orange dots). In all five cases, the complex band of the spectrum intersects the band of purely imaginary eigenvalues $[-i, i]$. (b) Eigenvalues located on the imaginary axis for $\alpha_{3}=1, \eta=0.0001$ and several values of $\rho \in[1,3]$.

both upper and lower halves of the complex plane are shown. As it can be seen from the figure, only the parabolic branch moves when the parameters change. Fig.6(a) is plotted for $\rho=2$ while Fig.6 (b) for $\rho=1$. In both cases, five spectra are shown for the parameter $\eta$ in the interval from 0.4 (orange dots) to 2 (red dots). Due to the symmetry of the solutions relative to the change of sign of $\eta$, the spectra are the same for negative values of $\eta$. The parabolic branch moves down when the parameter $\eta$ decreases.

When $\eta \rightarrow 0$, the parabolic branch in Fig.6 reduces to a single point in the imaginary axis. This limit corresponds to a KM soliton as indicated in Fig.1(b). The corresponding eigenvalue is given by $\lambda=i \sqrt{\rho}$. Fig.7(b) shows the IST spectrum of imaginary eigenvalues in this case. The upper isolated dot in the spectrum is the eigen- 
value of the KM soliton. It is perfectly described by the curve $\sqrt{\rho}$, which is represented by the blue dashed curve. When $\rho=1$, the KM soliton turns into the Peregrine solution with $\lambda=i$.

The branch of complex eigenvalues crosses the real axis when $\rho<1$. Fig.8(a) and 8(b) show the pattern of eigenvalues plotted for $\rho=0$ and $\rho=-1$ respectively. The five curves are calculated for the same set of $\eta$ values as in Fig.6. Each of these branches has mirror reflection symmetry with both $\mathrm{x}$ - and y-axis. Each branch has an upper limit that depends on the parameters of the solution.
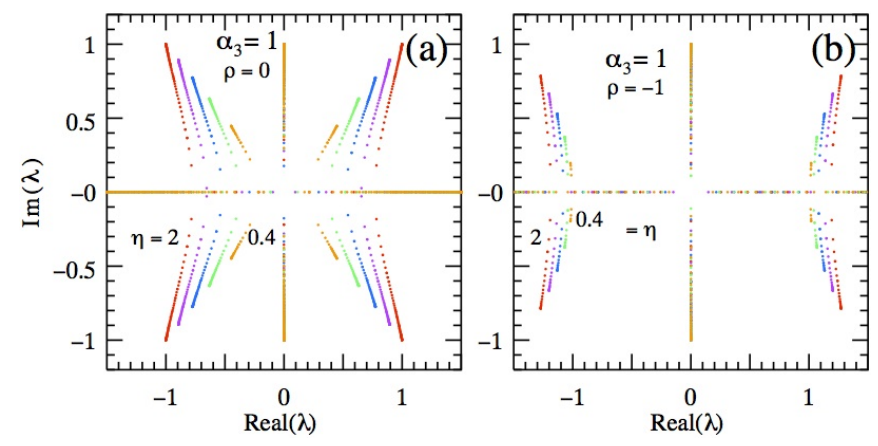

FIG. 8. IST spectra of five A-type doubly periodic solutions, corresponding to $\alpha_{3}=1$, (a) $\rho=0$, and (b) $\rho=-1$. The five curves are obtained for $\eta=2$ (red dots), $\eta=1.6$ (violet dots), $\eta=1.2$ (blue dots), $\eta=0.8$ (green dots) and $\eta=0.4$ (orange dots).

The branch of purely imaginary eigenvalues is related to the CW component of the solution. It coincides with the spectrum of ABs. We have not found a band gap as in the case of B-type solutions. The complex eigenvalues of the parabolic branch correspond to fixed amplitude waves propagating to the left or to the right with nonzero velocity. The symmetry of the eigenvalues in the left and the right halves of the complex plane provide the symmetry of the waves propagating to the left and to the right. Their interaction results in the standing oscillating wave which is the doubly periodic solution.

\section{CONCLUSIONS}

We have calculated, numerically, the IST spectral portraits of three-parameter families of doubly periodic solutions of the NLSE. They comprise two qualitatively different species that can be classified as of A- or B-type, depending on the presence or absence of a transverse displacement every half-period along the evolution variable.

We have shown that these two types of doubly periodic solutions have distinctively different IST spectral portraits. The IST eigenvalues for B-type solutions are located on the imaginary axis of the complex plane in the interval $[0, i]$. There is a band gap in this interval. Its width depends on the parameters of the doubly periodic solution. On the other hand, the imaginary IST eigenvalues for A-type solutions are located on the same interval but without a band gap. Instead, there is an additional band of complex eigenvalues. The location and limits of this band also depend on the parameters of the doubly periodic solution. In both cases, there is a continuous band of purely real eigenvalues that corresponds to small amplitude radiation waves.

This difference shows that there are not only mathematical but also physical differences between the two types of solutions. Type-A solutions can be considered as standing waves of nonlinearly interacting pairs of waves propagating in opposite directions along the transverse axis. This is not the case for B-type solutions. The latter are found to be incompatible with $\mathrm{ABs}$ of certain frequency range.

The work of JMSC is funded by Spanish MICINN grant RTI2018-097957-B-C33, and Comunidad de Madrid grant S2018/NMT-4326 SINFOTON2-CM
[1] A. R. Osborne, Nonlinear Ocean Waves and the Inverse Scattering Transform (Elsevier, 2010).

[2] V. E. Zakharov and A. B. Shabat, Exact theory of two-dimensional self-focusing and one-dimensional selfmodulation of waves in nonlinear media, Sov. Phys. JETP 34, 62 - 69 (1972).

[3] R. K. Dodd, J. C. Eilbeck, J. D. Gibbon, and H. C. Morris. Solitons and Nonlinear Wave Equations. (Academic Press, London, 1982).

[4] M. J. Ablowitz, D. J. Kaup, A. C. Newell \& H. Segur, The Inverse Scattering Transform-Fourier Analysis for Nonlinear Problems, Studies in Applied Mathematics. 53, 249 (1974).

[5] E. G. Turitsyna, S. K. Turitsyn, Digital signal processing based on inverse scattering transform, Opt. Lett., 38 (20), 4186 - 4188, (2013).

[6] S. K. Turitsyn, J. E. Prilepsky, S. T. Le, S. Wahls, L. L.
Frumin, M. Kamalian, and S. A. Derevyanko, Nonlinear Fourier transform for optical data processing and transmission: advances and perspectives, Optica, 4, $307-322$ (2017).

[7] L. L. Frumin, A. A. Gelash, and S. K. Turitsyn, New approaches to coding information using inverse scattering transform, Phys. Rev. Lett., 118, 223901 (2017).

[8] S. Randoux, P. Suret \& Gennady El, Inverse scattering transform analysis of rogue waves using local periodization procedure, Sci. Rep., 6, 29238 (2016).

[9] I. S. Chekhovskoy, O. V. Shtyrina, M. P. Fedoruk, S. B. Medvedev, and S. K. Turitsyn, Nonlinear Fourier Transform for Analysis of Coherent Structures in Dissipative Systems, Phys. Rev. Lett. 122, 153901 (2019).

[10] B. Deconinck, B. L. Segal, The stability spectrum for elliptic solutions to the focusing NLS equation. Physica D 346, 1-19 (2017). 
[11] E.D. Belokolos, A. I. Bobenko, V. Z. Enol'skii, A. R. Its, V. B. Matveev, Algebro-geometric approach to nonlinear integrable equations. (Springer, Berlin, 1994).

[12] G. Biondini \& G. Kovacic, Inverse scattering transform for the focusing nonlinear Schrödinger equation with nonzero boundary conditions, J. Math. Phys., 55, 031506 (2014).

[13] P. G. Grinevich \& P. M. Santini, The finite gap method and the analytic description of the exact rogue wave recurrence in the periodic NLS Cauchy problem, Nonlinearity 31, 5258 - 5308 (2018).

[14] M. Bertola, \& P. Giavedoni, A degeneration of two-phase solutions of the focusing nonlinear Schrödinger equation via Riemann-Hilbert problems, J. Math. Phys. 56, 061507 (2015).

[15] A. R. Its \& V. P. Kotlyarov, Explicit formulas for solutions of a nonlinear Schrödinger equation, Dokl. Akad. Nauk Ukr. SSR, Ser. 11, 965-968 (1976).

[16] F. Bonnefoy, A. Tikan, F. Copie, P. Suret, G. Ducrozet, G. Prabhudesai, G. Michel, A. Cazaubiel, E. Falcon, G. El, \& S. Randoux, From modulational instability to focusing dam breaks in water waves, Phys. Rev. Fluids 5, 034802 (2020).

[17] J. M. Soto-Crespo, N. Devine, \& N. Akhmediev, Integrable Turbulence and Rogue Waves: Breathers or Solitons ?, Phys. Rev. Lett. 116, 103901 (2016).

[18] Chong Liu, Zhan-Ying Yang, Wen-Li Yang, \& N. Akhmediev, Chessboard-like spatio-temporal interference patterns and their excitation, J. Opt. Soc. Am. B, 36, 1294 (2019).

[19] N. Akhmediev, V. M. Eleonskii, \& N. E. Kulagin, Exact first-order solutions of the nonlinear Schrödinger equation, Theor. Math. Phys. 72, 809-818 (1987).
[20] M. Conforti, A. Mussot, A. Kudlinski, S. Trillo, \& N. Akhmediev, Doubly periodic solutions of the focusing nonlinear Schrödinger equation: Recurrence, period doubling, and amplification outside the conventional modulation-instability band, Phys. Rev. A 101, 023843 (2020).

[21] N. Akhmediev, V. I. Korneev, N. V. Mitskevich, Nmodulation signals in a single-mode optical waveguide under nonlinear conditions. Sov. Phys. JETP, 67, $89-$ 95 (1988)

[22] F. Verhulst, Perturbation analysis of parametric resonance. In: R. Meyers (Eds), Encyclopedia of complexity and systems science. (Springer, New York, NY, 2009).

[23] N. Akhmediev, J. M. Soto-Crespo, A. Ankiewicz, Extreme waves that appear from nowhere: On the nature of rogue waves, Phys. Lett., A 373, 2137 - 2145 (2009).

[24] Y.-C. Ma \& M. J. Ablowitz, The Periodic Cubic Schrödinger Equation, Stud. Appl. Math. 65, 113-158 (1981).

[25] E. R. Tracy, H. H. Chen, \& Y. C. Lee, Study of quasiperiodic solutions of the nonlinear Schrödinger equation and the nonlinear modulational instability, Phys. Rev. Lett. 53, 218-221 (1984).

[26] N. Akhmediev and V. I. Korneev, Modulation instability and periodic solutions of the nonlinear Schrödinger equation, Theor. Math. Phys. 69, 1089-1093 (1986).

[27] N. Akhmediev and A. Ankiewicz, Solitons, nonlinear pulses and beams, (Chapman \& Hall, London, 1997), Chapter 13.

[28] P. F. Byrd and M. D. Friedman, Handbook of elliptic integrals for engineers and physicists, Springer (1954).

[29] M. Abramowitz and I. A. Stegun, Handbook of Mathematical Functions, Dover Publications (1974). 